\title{
Angle- and spin-resolved photoelectron spectroscopy in rotationally resolved photoionization of $\mathrm{HI}$
}

\author{
A. Mank *, M. Drescher, A. Brockhinke, N. Böwering, U. Heinzmann \\ Fakultät für Physik, Universität Bielefeld, D-33501 Bielefeld, Germany \\ Received: 5 October 1993/Final version: 29 December 1993
}

\begin{abstract}
The method of angle- and spin-resolved photoelectron spectroscopy has been used in combination with a laser based source of vacuum ultraviolet (VUV) radiation to study the photoionization dynamics of the HI molecule. The narrow bandwidth $\left(\Delta v<1 \mathrm{~cm}^{-1}\right)$ of the ionizing VUV radiation $\left(v=83200 \mathrm{~cm}^{-1}-89300 \mathrm{~cm}^{-1}\right)$ enables the resolution of the molecular rotation for single-photon excitation to spin-orbit autoionization resonances. The experimental results for the dynamical parameters $\sigma, A, \beta, \xi$, and $\alpha$ are compared to the results of a recent ab-initio calculation (M. Büchner, G. Raseev, and N.A. Cherepkov, J. Chem. Phys. 96, 2696 (1992)) and used to analyze the photoionization process in terms of partial contributions of different values of $l$ and $\lambda$ to the outgoing electron waves.
\end{abstract}

PACS: $33.20 . \mathrm{Ni} ;$ 33.60.Cv; 33.80.Eh

\section{Introduction}

Photoionization of atoms and molecules is one of the fundamental processes in the interaction of radiation with matter [1]. Studies of molecular systems are inherently more difficult than those of atomic systems, since additional degrees of freedom are involved. Detailed investigations of the dynamics of the molecular photoionization process were greatly assisted by the development of the laser. Since the ionization potentials for most small molecules lie above $10 \mathrm{eV}$, resonantly enhanced multiphoton ionization techniques (REMPI) have been developed [2]. Selecting the initial state of the photoionization process by a resonant excitation to a discrete state simplified the resulting spectra very much. However, due to a possible alignment induced in the intermediate state, and due to complications caused by several interacting

\footnotetext{
* Present address: Department of Chemistry, University of Waterloo, Waterloo, Ontario, N2L 3G1, Canada
}

or by dissociative intermediate states, the interpretation of the results can be difficult. Furthermore, not all molecular systems have suitable intermediate states for a resonant excitation process.

A different approach to the detailed study of molecular photoionization dynamics became possible with the development of frequency mixing of visible and ultraviolet (uv) laser radiation in metal vapors and noble gases [3]. Four-wave frequency mixing of dye laser beams produces coherent radiation in the spectral region from $200 \mathrm{~nm}$ to $70 \mathrm{~nm}$ with bandwidths of typically $1.0 \mathrm{~cm}^{-1}$, or even less, and photon intensities of $I \approx 10^{10}-10^{13} /$ pulse [4]. The narrow bandwidth of the vacuum ultraviolet (VUV) radiation enables the resolution of rotational structure for many small molecules in the excitation step. If combined with the technique of pulsed-field ionization with zero kinetic energy electron spectroscopy (PFIZEKE) [5], this method has proven to be a superb tool for the determination of ionization potentials of molecular ions. The resolution of the kinetic energy spectrum of the photoelectron is essentially limited only by the bandwidth of the radiation. However, the nature of the PFI-ZEKE technique restricts its application to the region of photoionization thresholds. In order to study the detailed dynamics of the autoionization process, more energetic electrons have to be detected. Here, rotational resolution in the kinetic energy spectrum of the photoelectron can be achieved only in exceptional cases [6], whereas vibrational structure can be resolved quite readily. In a generalized photoionization experiment, not only the dependence of the photoelectron emission on the excitation energy can be studied, but also the kinetic energy of the electron, its angle of emission and the orientation of the spin-polarization vector in the laboratory frame of reference [7]. The number of independent observables depends on the polarization of the ionizing radiation. In atoms, the higher spherical symmetry leads to the selection rule for the angular momentum of the photoelectron and the total angular momentum of the system

$\Delta \ell= \pm 1$ and $\Delta J=0, \pm 1$ 
respectively, which restricts the number of dipole matrix elements to be considered in the analysis of an experiment on closed shell systems to three. In this case, five independent observables prove to be sufficient for a quantum mechanically complete description of the photoionization experiment [8]. In molecules, this is generally not possible.

The high spectral brightness and the selectable polarization of the VUV radiation generated by frequency mixing enables for the first time the study of all dynamical parameters of the photoionization process resolving the rotational structure in the excitation step. It has been demonstrated for the asymmetry parameter $\beta$ of the angular distribution of the photoelectrons $[9,10]$, and a first experiment studying the influence of molecular rotation on the photoelectron spin polarization has been performed by Huth-Fehre et al. [11]. In this experiment, the angle-integrated photoelectron spin polarization was observed in spin-orbit autoionization to the ionic groundstate of the $\mathrm{HI}$ molecule.

Among the hydrogen halides $(\mathrm{HX}, \mathrm{X}=\mathrm{F}, \mathrm{Cl}, \mathrm{Br}, \mathrm{I})$, $\mathrm{HI}$ has the lowest ionization potential $(10.386 \mathrm{eV})$ [12]. This value was determined for a rotational temperature of $300 \mathrm{~K}$. A careful analysis of our experimental results in the threshold region at a rotational temperature of $13 \mathrm{~K}$ yields a value of $83738 \pm 8 \mathrm{~cm}^{-1}$ for the lowest rotational level $\left(\mathrm{J}^{+}=3 / 2\right)$ of the ionic ground state [13]. HI can be ionized by VUV radiation generated very efficiently in a mercury heat pipe [14]. The photoionization of an electron from the highest, non-bonding $\pi$-orbital leads to a $(p \pi)^{3}$ configuration and a ${ }^{2} \Pi$ ionic ground state. The strong spin-orbit interaction gives rise to a splitting of $5378 \pm 8 \mathrm{~cm}^{-1}$ [13] between the electronic term values of the two components of the ${ }^{2} \Pi$ state. Several Rydberg series converging to the higher lying ${ }^{2} \Pi_{1 / 2}$ component autoionize through spin-orbit interaction into the ${ }^{2} \Pi_{3 / 2}$ continuum. The large rotational constant of the neutral molecule $\left(B_{e}^{\prime \prime}=6.426 \mathrm{~cm}^{-1}\right)[15]$ and the similar value for the molecular ion $\left(B_{e}^{+} \approx 6.3 \mathrm{~cm}^{-1}\right)$ [16] lead to a simple rotational structure in the ionization spectra. This structure can be resolved quite readily in the excitation step using narrow-band VUV radiation generated by frequency mixing.

Previous angle- and spin-resolved experiments on photoelectrons from HI using synchrotron radiation have concentrated on the photon energy region above the ${ }^{2} \Pi$ ionic thresholds, investigating the influence of electronic autoionization and the direct transition to the open continuum [17]. Polarization measurements were also carried out with resolution of the molecular vibration in the final ionic states and it was shown that specific information on the coupling of resonances to the outgoing partial electron waves can be obtained by combining cross section and polarization data [18]. However, in such studies of the energy dependence of the spin-polarization parameters at a resolution of $\Delta \lambda=0.5 \mathrm{~nm}$, only broad structures can be analyzed.

Several theoretical studies covering different aspects of the photoionization of the HI molecule are available for the spin-orbit autoionization region $[16,19,20,21]$. After the first multichannel quantum-defect calculation of the polarization parameters [19] a formalism which in- cludes the molecular rotation explicitly was developed by Raseev and Cherepkov [20]. This theory was then applied to a calculation using pure Hund's case (e) coupling and compared with available experimental cross section and spin polarization results [21]. Lefebvre-Brion has then extended the calculations to include a transition in angular momentum coupling from Hund's case $(c)$ to $(e)$ which gave improved agreement with experimental total yield results for Rydberg orders from 10 to 14 [16].

The purpose of this paper is to report on an extension of the previous rotationally resolved studies on HI $[9,11,16,22]$ by performing now angle-resolved measurements of the spin polarization components to obtain the dynamical parameters $A, \alpha$, and $\xi$. Furthermore, results for the asymmetry parameter $\beta$ of the photoelectron angular distribution were obtained for the same expansion conditions of the molecular beam and the measurements of the angle-integrated spin polarization were extended to higher photon energies.

\section{Experimental}

The experimental arrangement for angle- and spin-resolved photoelectron spectroscopy on small molecules can be divided into two parts: the generation and analysis of the polarized VUV radiation and the photoionization experiment with subsequent energy, angle, and spin resolved measurements of the emitted photoelectrons.

The ionizing VUV radiation is generated by resonantly enhanced four-wave sum-frequency mixing of pulsed dye laser radiation in mercury vapor. A detailed description of the experimental setup for the generation of the VUV radiation has been given elsewhere [22]. Briefly, it consists of two dye lasers pumped by the frequency doubled output of a Nd: YAG laser. The output of one dye laser is frequency doubled in a $\mathrm{KD}^{*} \mathrm{P}$ crystal and tuned to the $6 d^{1} D_{2} \leftarrow 6 s^{1} S_{0}$ two-photon resonance in mercury at $71333.18 \mathrm{~cm}^{-1}(\lambda \sim 280.3 \mathrm{~nm})$ for a resonant enhancement of the mixing process. The output of the second dye laser is overlapped with this uv radiation in a dichroic mirror and both beams are focused $(f=1000 \mathrm{~mm})$ into the vapor zone of a mercury heat-pipe operating at approximately $7 \mathrm{mbar}$ of $\mathrm{Hg}$-pressure with $\sim 13 \mathrm{mbar}$ of $\mathrm{Ne}$-gas as a buffer. A telescope in the path of the visible dye laser adjusts the focal conditions for optimum VUV generation. Tuning the visible dye laser from $\lambda=560 \mathrm{~nm}$ to $\lambda=840 \mathrm{~nm}$ results in VUV generation in the energy region from $89300 \mathrm{~cm}^{-1}$ to $83200 \mathrm{~cm}^{-1}$. The bandwidth of the VUV radiation $\left(<1.0 \mathrm{~cm}^{-1}\right)$ is determined by the bandwidth of the incoming dye laser radiation.

A LiF prism separates the generated radiation at the sum frequency from the incoming laser radiation and other mixing products [14], and steers the beam onto the photoionization target. Behind the target region, a vacuum photodiode monitors the intensity of the VUV signal. This photodiode can be rotated out of the beam to allow the VUV radiation to pass through a polarization analyzer. The analyzer is of the reflecting surface type using four gold-coated mirrors [23]. After passing through the analyzer, the VUV beam impinges on a copper plate 
at normal incidence. The emitted photoelectrons are deflected and detected by an electron multiplier mounted at an angle of $90^{\circ}$ from the copper electrode. This arrangement allows the polarization analysis of the VUV radiation without unwanted polarization dependent effects from the use of tilted surfaces for the detection of the VUV intensity [24]. When the copper electrode is removed from the VUV beam path, the radiation is incident on a multi-channel plate (MCP) with a phosphor screen to visualize the beam. This image converter proved to be very useful in achieving a good alignment of the VUV beam onto the photoionization target, which is essential for the measurement of the photoelectron angular distribution and the spin polarization.

The fundamental dye laser radiation is linearly polarized with a high degree of polarization $(P>0.98)$. Since the linear polarization of the frequency doubled radiation is oriented at a right angle to the fundamental laser radiation, the polarization of the second dye laser was rotated by $90^{\circ}$. Having the polarization vectors of both incoming frequencies parallel to each other ensures a high degree of linear polarization of the VUV radiation. The orientation of the VUV polarization can be rotated around the propagation direction by rotating the polarization of both incoming laser beams simultaneously. This was achieved by inserting a half-wave Fresnel rhomb into the combined beams in front of the lens (Fig. 1a). This scheme is preferred for measurements of photoelectron angular distributions $[9,10]$ and the component of the photoelectron spin polarization perpendicular to the reaction plane [25]. In the first studies of the angle-integrated photoelectron spin polarization $[11,22]$ a thin $\mathrm{MgF}_{2}$ quarter-wave plate was used to achieve circular polarization of the VUV radiation. The phase shift of this plate is strongly frequency dependent and had to be angle- and temperature-tuned. The transmission of the plate $\left(50 \%\right.$ at $\left.86250 \mathrm{~cm}^{-1}\right)$ decreases rapidly when the frequency of the VUV radiation is further increased.

For the studies reported in this paper, we have chosen a different way to generate the circularly polarized VUV radiation: a so-called achromatic quarter-wave plate consisting of a quartz plate and a $\mathrm{MgF}_{2}$ plate $(\lambda / 4$ at $280.3 \mathrm{~nm}$ and $\lambda / 4 \sim 650 \mathrm{~nm}$, Halle Optik, Berlin) was inserted into the combined laser beams before the lens. This plate induces a phase shift in the dye laser beams. With the linear polarization of both laser beams parallel to each other, both beams are circularly polarized with the same helicity after passing through the quarter-wave plate. In this case, no radiation is observable at the sum-frequency as expected from similar results for third harmonic generation [26]. By rotating the quarter-wave plate by $45^{\circ}$, no phase shift is introduced to the passing laser beams, thus linearly polarized VUV radiation is generated. This result is useful for consistency checks while measuring the photoelectron spin polarization. By inserting the half-wave Fresnel rhomb into the beam of the visible dye laser before the dichroic mirror, its linear polarization vector can be rotated independently of the orientation of the polarization vector of the second laser. Orienting the linear polarization of the incoming beams at right angles with respect to each other yields circularly polarized laser radiation
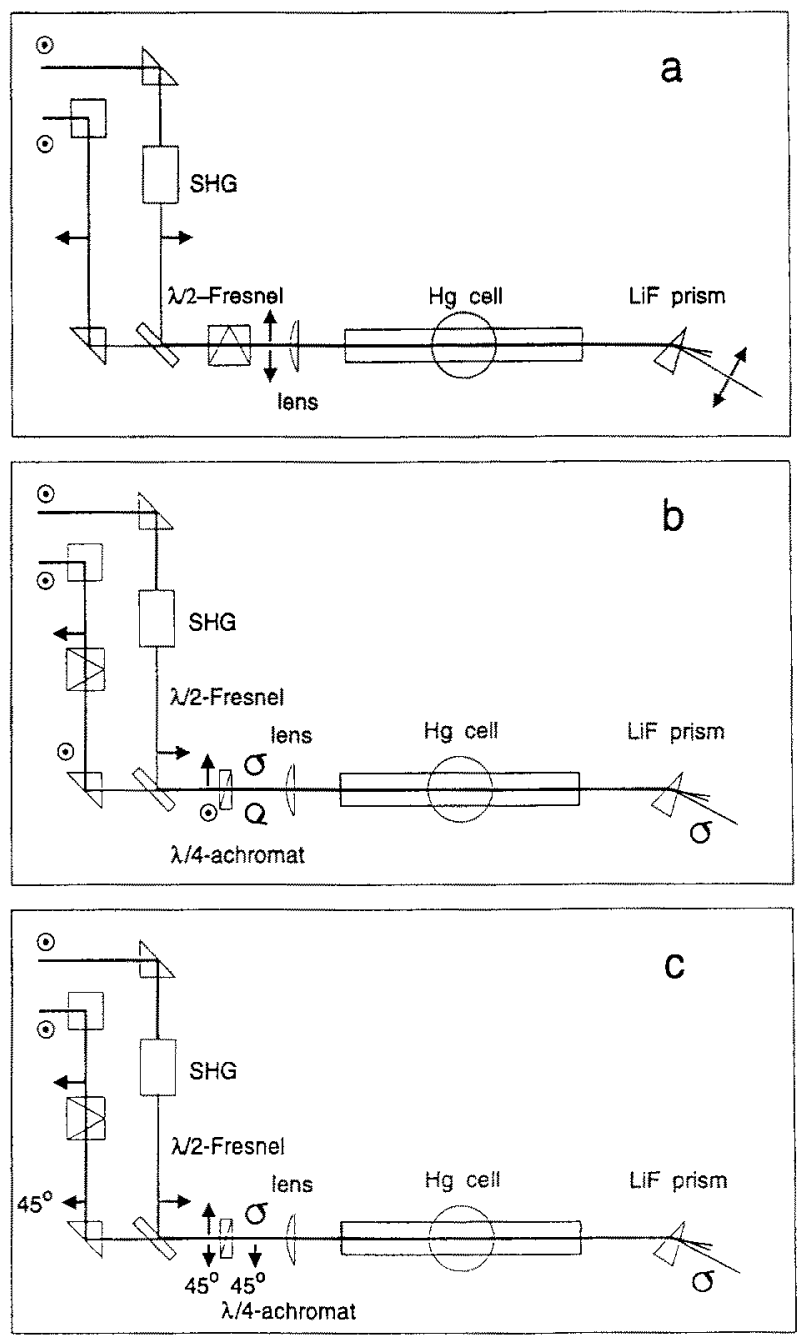

Fig. 1a-c. Schematic view of the setup for generation of polarized VUV radiation. a Optical setup for linearly polarized VUV, where the orientation of the VUV polarization is selectable by rotating the polarization vectors of the incoming laser radiation. $b$ Optical setup for circularly polarized VUV radiation by circularly polarizing the incident laser radiation. The achromatic $\lambda / 4$-plate requires the linear polarizations of the two dye laser beams to be perpendicular to each other. $\mathbf{c}$ The polarization of the visible dye laser is oriented at an angle of $45^{\circ}$ to the polarization of the uv radiation. After passing through the achromatic wave plate, the uv radiation is circularly polarized, the visible radiation remains linearly polarized. The resulting VUV radiation is circularly polarized

of opposite helicity for the two frequencies (Fig. 1b). The resulting helicity of the VUV light is then determined by the helicity of the uv radiation tuned to the two-photon resonance.

Another scheme for generating circularly polarized VUV radiation was tested in the following way: the halfwave Fresnel rhomb was used to rotate the linear polarization of the visible laser to an angle of $45^{\circ}$ with respect to the linear polarization of the uv radiation. This results in circularly polarized uv radiation and linearly polarized visible radiation after passing through the quarter-wave plate (Fig. 1c). The polarization of the VUV radiation produced is again circular, but the intensity is reduced 
by a factor of two. This can be explained by photon angular momentum conservation. Since linearly polarized radiation can be described as a mixture of right- and left-handed polarization with equal intensities, only the part with the correct polarization is involved in the VUV generation process, reducing the intensity by a factor of two. This result will be useful for future experiments in different regions of VUV energies. It is not necessary to obtain a set of achromatic quarter-wave plates tailored to each mixing process, since it is possible to generate circularly polarized radiation with just a quarter-wave plate for the resonant laser frequency.

The photoionization target is prepared by a free jet expansion of the target gas through a pulsed nozzle. The nozzle (Lasertechnics LPV) employs a piezoelectric plate for the movement of the valve, thereby minimizing stray electromagnetic fields which could influence the angular distribution and the spin polarization of the low-energy $\left(E_{\text {kin }}<1 \mathrm{eV}\right)$ photoelectrons. The HI gas was purchased from Merck-Schuchardt with a stated purity of $98 \%$ and used without further purification. The gas-handling system was heated to $50^{\circ} \mathrm{C}$ to prevent built-up of iodine crystallites. No traces of iodine, which has a lower ionization potential than HI, could be detected in the photoelectron spectrum. The expansion of $150 \mathrm{mbar}$ neat $\mathrm{HI}$ through a $300 \mu \mathrm{m}$ orifice leads to a rotational temperature of approx. $13 \mathrm{~K}$. This value can be deduced from the rotational structure observed in the photoionization spectrum $[11,16]$ and from results of $1+1$ REMPI via the $b^{3} \Pi_{1}$ state [22].

The results reported in this paper were obtained with three different detection systems for the emitted photoelectrons. The total photoionization yield and the angleintegrated spin-polarization have been measured using an electrostatic extraction field which has been described in detail elsewhere $[11,27]$. The photoelectron angular distributions, characterized by their asymmetry parameter $\beta$, have been measured with an electrostatic energy analyzer. The instrument is a simulated hemispherical analyzer [28] which gives a best resolution of $60 \mathrm{meV}$ and was typically operated at a bandpass of $100 \mathrm{meV}$. This is sufficient to separate the different vibrational channels of the molecular ion $\left(\Delta E_{\mathrm{vib}} \sim 250 \mathrm{meV}\right)$ [29]. The angular resolution $\left(\Delta \theta \sim 4^{\circ}\right)$ is given by an aperture in the lens system used to image the target onto the entrance slit of the analyzer. The electrons are detected by a two-stage MCP mounted behind the exit slit. The angular distributions were recorded by keeping the analyzer fixed in space and rotating the linear polarization of the VUV through the reaction plane. Details of this experimental setup have been published elsewhere $[9,30]$.

Before presenting the setup for the angle- and spinresolved photoionization experiment, the angular dependence of the different observables will be discussed. For linearly polarized radiation, the following relations hold for the differential cross section [31] and the spin polarization component perpendicular to the plane of reaction $P_{\perp}(\theta)[32]$ :

$\frac{d \sigma}{d \Omega}(\theta)=\frac{\sigma}{4 \pi}\left[1+\beta P_{2}(\cos \theta)\right]$
$P_{\perp}(\theta)=-\frac{4 \cdot \xi \cos \theta \sin \theta}{1+\beta P_{2}(\cos \theta)}$

where $P_{2}(\cos \theta)$ is the second Legendre polynomial,

$P_{2}(\cos \theta)=\frac{3}{2} \cos ^{2} \theta-\frac{1}{2}$.

$\theta$ is the reaction angle between the direction of the linear polarization vector of the incident radiation and the momentum of the photoelectron. $\sigma$ denotes the photoionization cross section for a given final state and $\beta$ the asymmetry parameter of the angular distribution. $P_{\perp}(\theta)$ is parameterized by $\xi$ and can be obtained by using the electron spectrometer in a space-fixed geometry analyzing the electrons with respect to their spin polarization in a Mott detector [33]. By rotating the polarization vector of the ionizing radiation to the so called 'magic angle', where $P_{2}(\cos \theta)=0\left(\theta=54.7^{\circ}\right)$, the parameter $\xi$ can be measured independent of the angular distribution of the photoelectron intensity.

More independent observables can be obtained from an experiment using circularly polarized radiation, where by convention the angle $\theta$ is defined between the momenta of the photon and the photoelectron. The relations given above have to be modified for circularly polarized radiation [34]:

$$
\begin{aligned}
& \frac{d \sigma}{d \Omega}(\theta)=\frac{\sigma}{4 \pi} F(\theta)=\frac{\sigma}{4 \pi}\left[1-\frac{\beta}{2} P_{2}(\cos \theta)\right] \\
& P_{\perp}(\theta)=P_{Y}(\theta)=2 \xi \frac{\sin \theta \cos \theta}{F(\theta)} .
\end{aligned}
$$

In addition, the other two Cartesian components of the spin-polarization vector in the plane of reaction which depend on the light helicity $\gamma$ are different from zero:

$$
\begin{aligned}
& A(\theta)=P_{Z}(\theta)=\frac{1}{F(\theta)}\left\{\gamma\left[A-\alpha P_{2}(\cos \theta)\right]\right\} \\
& P_{p}(\theta)=P_{X}(\theta)=-\gamma \frac{3}{2} \alpha \frac{\sin \theta \cos \theta}{F(\theta)} .
\end{aligned}
$$

$\alpha$ is the angular asymmetry parameter of the spin polarization, $A(\theta)$ is the component parallel to the photon spin. This component is the only one which does not vanish after integrating over all emission angles [34].

For angle-resolved determination of the spin-polarization component $A(\theta)$ and the parameter $\alpha$, the electron analyzer has to be rotated through the reaction plane and, subsequently, the electrons have to be transported to the space-fixed Mott detector for spin polarization analysis. This was realized with an electron lens system similar to the one used in experiments with synchrotron radiation [35]. The setup for these measurements is shown in Fig. 2. After passing through the spectrometer, the electrons are accelerated towards a $90^{\circ}$ deflector. Behind the deflector the electron path is parallel with the axis of rotation for the energy analyzer. A four-element zoom lens images the electrons from the exit of the first detector onto the entrance of a second $90^{\circ}$ deflector. Thereby, the electrons are transferred to a frame with fixed axis in- 


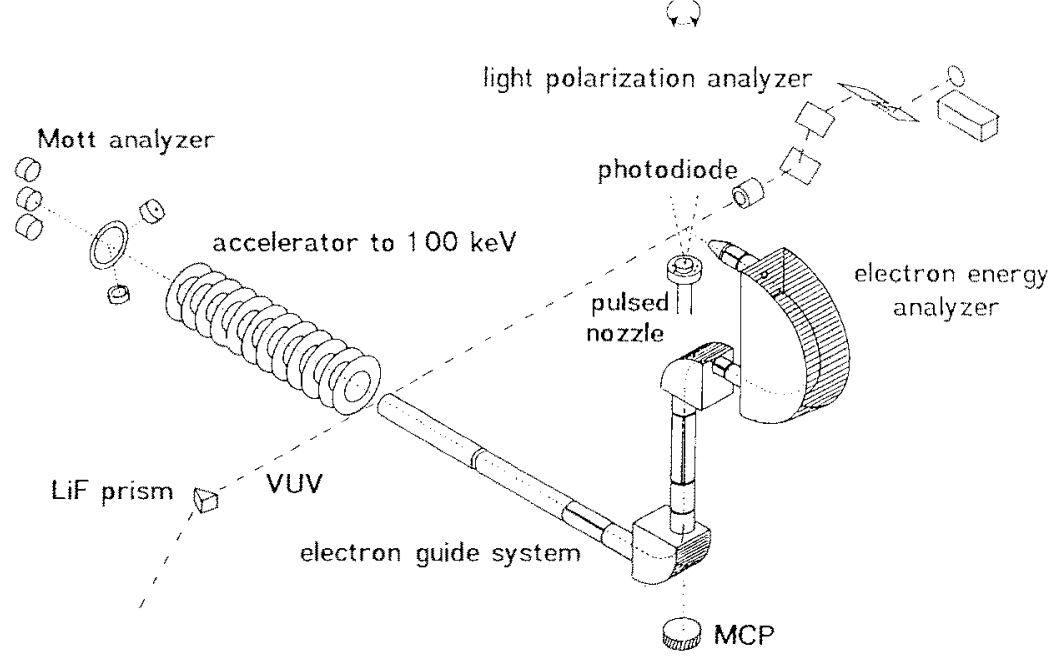

Fig. 2. Schematic view of the photoionization experiment. The photoelectrons produced in the ionization region located $4 \mathrm{~cm}$ above the pulsed nozzle are detected angle-resolved by an electron spectrometer rotatable in the plane of reaction given by the momentum of the photoelectron and the ionizing VUV radiation. Two $90^{\circ}$ deflectors are used to transfer the electrons onto a space-fixed axis irrespective of their emission direction. The spin polarization of the photoelectrons is inferred from the asymmetric count rates produced by Mottscattering at $100 \mathrm{keV}$ in the two backscattering detectors dependent of the angle of the electron energy analyzer with respect to the photon momentum. A MCP detector mounted on the axis of rotation is used to monitor the alignment of the electron beam in the lens system. After passing through the second $90^{\circ}$ deflector, another fourelement zoom lens accelerates the electrons to $3.5 \mathrm{keV}$. With this energy, they enter a 12 -fold linearly divided acceleration stage where they reach a kinetic energy of $100 \mathrm{keV}$. The electrons are then scattered at a thin gold foil (thickness $\sim 1000 \AA$ ). The transverse component of the spin polarization of the electrons can be detected by measuring the scattering asymmetry in the backward direction at scattering angles of $120^{\circ}$ [33]. Additional detectors to record the direct beam and the forward scattering are mounted on axis and at $\pm 13^{\circ}$. They are used to measure the photoelectron intensity and to compensate for instrumental asymmetries. The electric potentials for the electron lenses are supplied by a single power supply, which is referenced to ground. A microcomputer (ATARI 1040 ST) interfaces through a bus system with a set of up to 127 active voltage dividers. A program, written in $\mathrm{C}$, calculates the correct settings referenced to ground from the settings referenced to the kinetic energy given by the operator and adjusts the voltage dividers accordingly.

The orientation of the reaction plane and the spinpolarization vector for these measurements is depicted in Fig. 3a. The polarization component perpendicular to the reaction plane, which is proportional to the parameter $\xi$, can be measured in two different ways. Using circularly polarized radiation, it could be determined simultaneously by employing a Mott detector with two complete sets (four each) of detectors in perpendicular planes. Alternatively, the Mott detector could be rotated by $90^{\circ}$, taking the measurements in series. In the present work, the perpendicular component of the photoelectron spinpolarization is, however, measured with linearly polarized light. The reaction plane and the polarization vector for this setup are depicted in Fig. 3b. Since the reaction
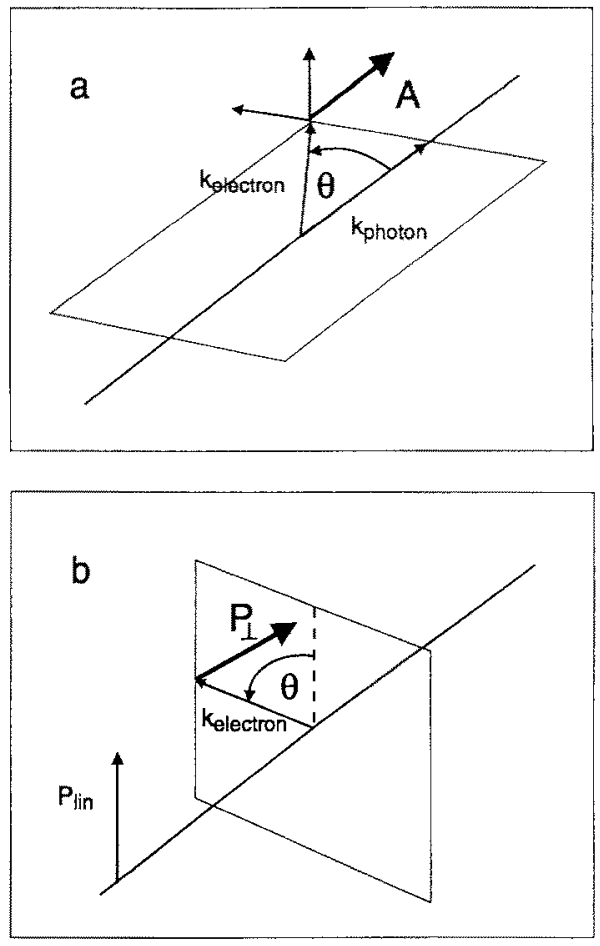

Fig. 3a, b. Schematic view of the two different experimental geometries. a The momentum vector of the incident circularly polarized radiation and the momentum vector of the outgoing photoelectron form the reaction plane. The spin polarization component $A(\theta)$ lies in the reaction plane, and its angular distribution is measured by rotating the electron detector. b The linear polarization of the incident radiation and the momentum vector of the outgoing photoelectron form the plane of reaction. The spin polarization component $P_{L}(\theta)$ is perpendicular to the plane of reaction. The angular distribution is measured by rotating the vector of the linear polarization of the ionizing radiation. In the laboratory, the direction of this component is identical to the direction of $A$ in the case of incident circularly polarized radiation 
plane is now given by the photon polarization vector and the electron momentum, it is rotated by $90^{\circ}$ with respect to the reaction plane for circularly polarized incident radiation. This way, the Mott analyzer does not have to be rotated to measure this component.

The intensity of the VUV radiation $\left(\sim 10^{10} /\right.$ pulse $)$ leads to electron signals of typically 50 electrons/pulse in an angle-resolved intensity or an angle-integrated spinpolarization experiment. Because of the short pulse length of the VUV radiation ( $\sim 4 \mathrm{~ns})$, these signals appear as pulses of similar length. The signals are measured by integrating the accumulated charge of the electrons in a charge-sensitive preamplifier (Canberra 1003). The pulse height of the output of the preamplifier is then sampled and digitized for storage in an microcomputer. In the experiments resolving the angular distribution and the spin polarization of the photoelectrons simultaneously, the signal strength dropped by a factor of 1000 , to typically one electron detected for 20 pulses. By carefully measuring the pulse-height distribution of the surface barrier detectors in the Mott analyzer, it is possible to distinguish between $0,1,2$ and even 3 electron events quite readily [36]. For these experiments, we therefore measured the actual numbers of electrons at the detector in a counting experiment, using the computer as a software discriminator.

\section{Results}

\subsection{VUV polarization}

Typical results for the measurement of the VUV polarization are shown in Fig. 4. For the measurements, the four-mirror analyzer is rotated in steps of $10 \mathrm{deg}$. around the propagation direction of the VUV radiation. At each angle, the intensity of the electron signal on the multiplier behind the analyzer is averaged over 50 laser shots. The angular dependence of the transmitted radiation is expected to be of the form [37]:

$I=I_{\min }+\left(I_{\max }-I_{\min }\right) * \cos ^{2} \phi$,

where $\phi$ is the angle of the polarizer with respect to a space-fixed axis, $I_{\max }$ and $I_{\min }$ denote the intensity maxima and minima, respectively, of the angular dependence. The experimental results for linearly polarized radiation are given in Fig. 4a. The error bars, indicating the statistical uncertainty of the averaging, are smaller than the point size. The full line represents the result of a least-squares fit to (8). From the fit, the values for $I_{\max }$ and $I_{\min }$ are determined, which then give the degree of linear polarization according to [38]:

$P_{\operatorname{lin}}=\frac{\left(I_{\max }-I_{\min }\right)}{\left(I_{\max }+I_{\min }\right)}$

This value for the linear polarization is given in Table 1 , line a and was obtained with the set up according to Fig. 1a. The result does not change by using the setup according to Fig. $1 \mathrm{~b}$ with the appropriate choices for the

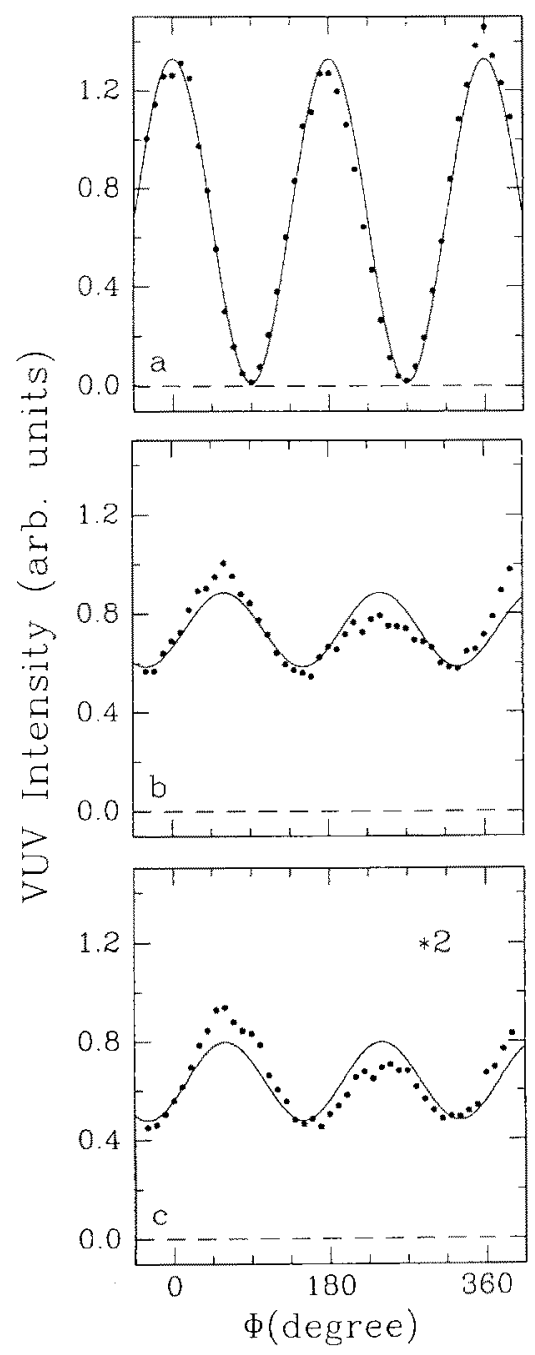

Fig. 4a-c. Experimental results for the polarization of the VUV radiation at a photon energy of $86885 \mathrm{~cm}^{-1}$. The error bars denoting the statistical uncertainty are smaller than the points depicting the results for the dependence of the transmitted intensity on the analyzer orientation. The lines drawn through the experimental data are the result of a least-squares fit to (10). The resulting values for the polarization are listed in Table 1 . An explanation of the different measurements is given in the text

orientation of both wave plates for the generation of linearly polarized radiation. In particular, the linear polarization is almost equal to unity if one takes the analyzing power of the four-mirror instrument with its theoretical maximum of 0.983 [23] at this VUV wavelength in account.

Assuming that rotation of the quarter-wave plate into the position of optimum phase shift does not introduce a depolarization action, the degree of circular polarization can be obtained from a similar measurement. It can be calculated from the constants $I_{\max }$ and $I_{\min }$ determined by the fit as follows [38]:

$P_{\mathrm{cir}}=\frac{2 \sqrt{I_{\max } * I_{\min }}}{I_{\max }+I_{\min }}$.

The results for the generation of circularly polarized radiation according to the schemes of Fig. $1 \mathrm{~b}$ and $\mathrm{c}$ are 
Table 1

\begin{tabular}{lll}
\hline $\mathrm{a}$ & $P_{\text {lin }}$ & $0.981 \pm 0.035$ \\
$\mathrm{~b}$ & $P_{\text {cir }}$ & $0.979 \pm 0.005$ \\
$\mathrm{c}$ & $P_{\text {cir }}$ & $0.968 \pm 0.009$ \\
\hline
\end{tabular}

shown in Fig. 4b and c, respectively. The results in Fig. 4c have been multiplied by a factor of two, in order to compensate for the intensity loss due to the fact that one laser is linearly polarized. The degrees of circular polarization determined by the fit to (8) and application of (10) are given in Table 1 , lines $b$ and $c$, respectively. The results for the two different schemes to produce circularly polarized radiation are identical within the uncertainty of the measurement.

\subsection{Photoionization experiment}

Since the degree of circular polarization is less than one, the influence of the polarization ellipse on the photoionization dynamics has to be considered. The general formulas for ionizing radiation characterized by the Stokes parameters $S_{1}, S_{2}$, and $S_{3}$ have been derived by Huang [39] and Cherepkov [40]. In our case, they can be further simplified:

$$
\begin{aligned}
\frac{d \sigma}{d \Omega}(\theta) & =\frac{\sigma}{4 \pi} F(\theta) \\
& =\frac{\sigma}{4 \pi}\left[1-\frac{\beta}{2} P_{2}(\cos \theta)-S_{1} \frac{3}{4} \beta \sin ^{2} \theta\right] \\
A(\theta)= & P_{Z}(\theta)=\frac{1}{F(\theta)} \\
& \times\left\{\gamma S_{3}\left[A-\alpha P_{2}(\cos \theta)\right]-S_{2} 2 \xi \sin ^{2} \theta\right\}
\end{aligned}
$$

where $\gamma$ is the helicity of the VUV radiation $(\gamma=+1$ for $\sigma^{+}$- and $\gamma=-1$ for $\sigma^{-}$-radiation). The Stokes parameters are defined as:

$S_{1}=P_{\text {lin }} \cos 2 \varphi$

$S_{2}=P_{\operatorname{lin}} \sin 2 \varphi$

$S_{3}=P_{\text {cir }}$.

Here, $\varphi$ is the angle of the major axis of the polarization ellipse with respect to the plane of reaction. The main effect of the weak admixture of linearly polarized light to the VUV radiation is that the influence of the angular distribution of the photoelectron intensity does not completely vanish for detection at the magic angle. In the experiments using synchrotron radiation $[17,34,35]$, this could be circumvented by choosing $\varphi=45^{\circ}$ so that $S_{1}=0$ independent of the ellipticity of the radiation. This was not possible in the experiments described here. Contrary to a synchrotron light source, the orientation of the polarization ellipse is not fixed. It can change due to the influence of optical elements in the light beam. Therefore, the asymmetry parameter $\beta$ had to be determined with the same laser source to enable the deconvolution of the influence of the angular distribution of the photoelectron intensity on the spin polarization measurements.
The polarization component perpendicular to the plane of reaction has been measured using linearly polarized radiation. The influence of the ellipticity of the VUV polarization reduces to a normalization factor for angle-integrated measurements. Hence, only for the measurements of the angular distribution of the in-plane component of the spin polarization (12), the effects of the polarization ellipse have to be considered. Inspection of (12) reveals, that for a change of the helicity of the radiation, the term proportional to $S_{3}$ changes sign. By measuring with both helicities and subtracting the data from each other, the term proportional to $S_{2}$ can be eliminated. Then, with the exact knowledge of the parameter $S_{1}$ and $\beta$, the result can be deconvoluted to the case of $100 \%$ circular polarization. Angular distributions of the spin polarization for the ${ }^{2} \Pi_{3 / 2}, v^{+}=0$ final ionic state were recorded in the intensity maxima of the 6th and 7 th Rydberg order at VUV energies of $85770 \mathrm{~cm}^{-1}$ and $86700 \mathrm{~cm}^{-1}$, respectively. The deconvoluted angular distributions are shown in Fig. 5. The change due to the deconvolution is on the order of $10-15 \%$ of the magnitude of the value. A least-squares fit of the data to equation 6 yields the values for the parameters $A$ and $\alpha$ given in Table 2.

In Fig. 6, all available results for the dynamical parameters in the photoionization of the HI molecule in the spin-orbit autoionization region at a rotational temperature of $13 \mathrm{~K}$ are shown. The results for the photoionization yield have been published before $[11,16]$ and are reproduced here to relate the resonance structure in the other parameters to the structure in the photoionization cross section. The results for the angle-integrated spin polarization parameter $A$ in the energy range of $85600 \mathrm{~cm}^{-1}$ to $86110 \mathrm{~cm}^{-1}$ and around $86300 \mathrm{~cm}^{-1}$, which have been published previously [11,22], are shown for completeness. All the experimental results are denoted by error bars. These indicate not only the statistical uncertainties due to averaging over a limited number of laser

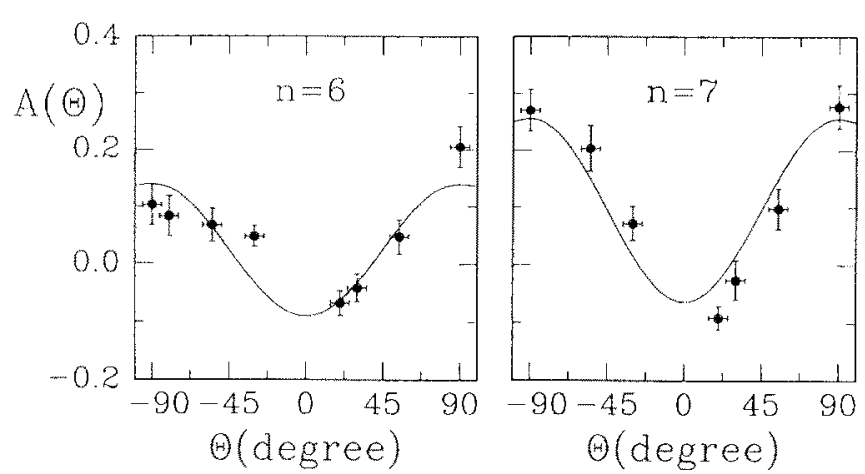

Fig. 5. Angular distribution of the spin polarization at $v=85770 \mathrm{~cm}^{-1}$ a and $v=86700 \mathrm{~cm}^{-1}$ b for the ${ }^{2} \Pi_{3 / 2}, v=0$ final ionic state. The experimental results are denoted by error bars, the lines are the result of a least-squares fit to (6) yielding the parameters $A$ and $\alpha$ listed in Table 2

Table 2

\begin{tabular}{lll}
\hline Energy $\left(\mathrm{cm}^{-1}\right)$ & $A$ & $\alpha$ \\
\hline 85770.0 & $0.064 \pm 0.017$ & $0.154 \pm 0.017$ \\
86700.0 & $0.150 \pm 0.021$ & $0.214 \pm 0.020$ \\
\hline
\end{tabular}




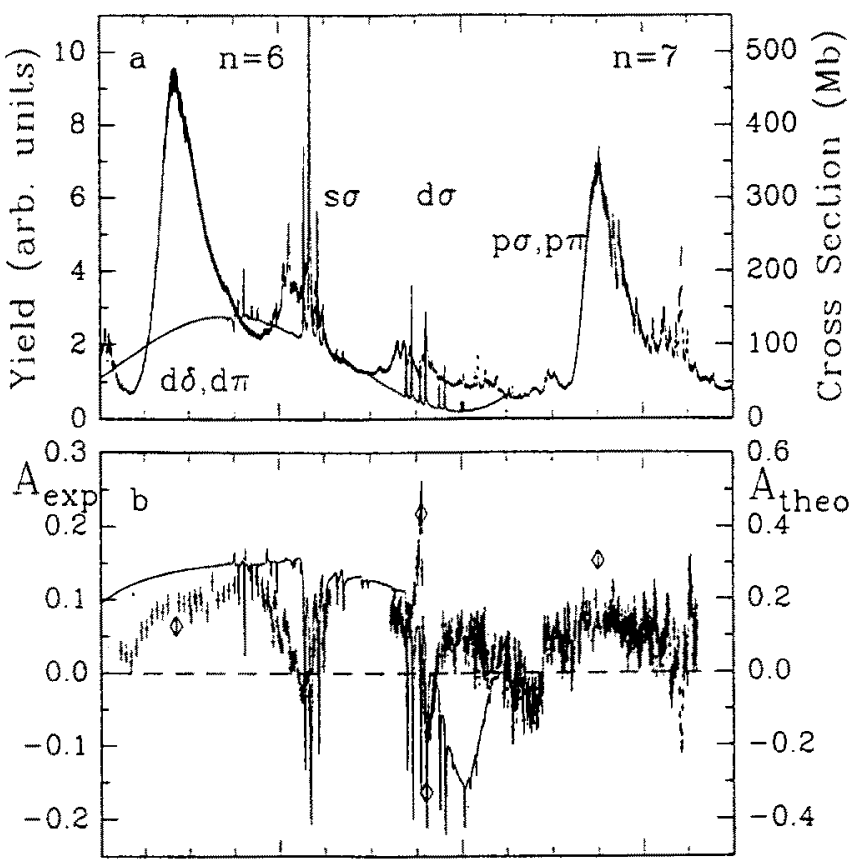

shots, but also include the error in determining the VUV polarization and the uncertainty of the analyzing power of the Mott detector (Sherman function: $-0.25 \pm 0.01$ ), where applicable. The results for the angle-integrated spin polarization are also energy-integrated over the two possible vibrational channels of the ion. To give an estimate for the influence of the vibration onto this parameter, four values of the parameter $A$ for the ${ }^{2} \Pi_{3 / 2}, v=0$ ionic channel alone are shown as large squares. Two of these were obtained from fits of the component $A(\theta)$, the other two were measured at the magic angle and the influence of the asymmetry parameter $\beta$ was deconvoluted.

The full-drawn line is the result of a calculation by Büchner et al. [21]. This calculation assumes a pure Hund's case $(e)$ angular momentum coupling and the influence of rotational autoionization is neglected. We are not able to determine absolute cross sections, and the overlap of the theoretical and the experimental results in Fig. 6a is arbitrary. The maximum of the calculation gives a cross section of $1024 \mathrm{Mb}$ for the $n^{*}=6, s \sigma, R(0)$ transition after convoluting the theoretical result to the experimental bandwidth of $\approx 1 \mathrm{~cm}^{-1}$. Carlson et al. [41] measured the cross section at the broad resonance at $85700 \mathrm{~cm}^{-1}$ to be $60 \mathrm{Mb}$, using synchrotron radiation with a bandpass of $0.07 \AA$.

\section{Discussion}

The resonances in the photoionization results of Fig. 6 are connected with states of the configuration $(5 p \pi)^{3} n l \lambda$ converging to the $\mathrm{HI}^{+} X^{2} \Pi_{1 / 2}$ limit and autoionizing to the open continuum. The experimental data cover the range of principal Rydberg order $n=6$ and 7 and the resonance can be assigned to broad $d$-, sharp $s$ - and $f$ and weak $p$ - and $g$-complexes [42]. This range of excitation energies was chosen for our first studies of the dynamical parameters after an initial study of the photoionization yield in the full energy range between the spin-orbit split ionic ground states revealed strong perturbations for $n=8$ and 9 and a change of angular momentum coupling cases in the region of $n>10$ [16]. Comparing the theoretical and the experimental results, one finds that nearly all the resonances are predicted to be much sharper and more pronounced than experimentally observed. This is clearly not an effect of limited resolution, since the sharpest structures to be found in the ex-

Fig. 6a-e. Comparison of the experimental results for the dynamical parameters with the results of an ab-initio calculation [19]. The experimental results are denoted by points with error bars. The results of the calculation, shown by a full line, are shifted by $111 \mathrm{~cm}^{-1}$ to lower energy for a match in the position of the $n=6$, $s \sigma, R(0)$ transition. The scaling of the theoretical results for the cross section to the experimental results of the photoelectron yield is arbitrary. In b the theoretical results for the $A$ parameter are presented on a scale reduced by a factor of two with respect to the experimental results and four experimental points for the ${ }^{2} \Pi_{3 / 2}$, $v=0$ channel are included for comparison with the energy integrated results 

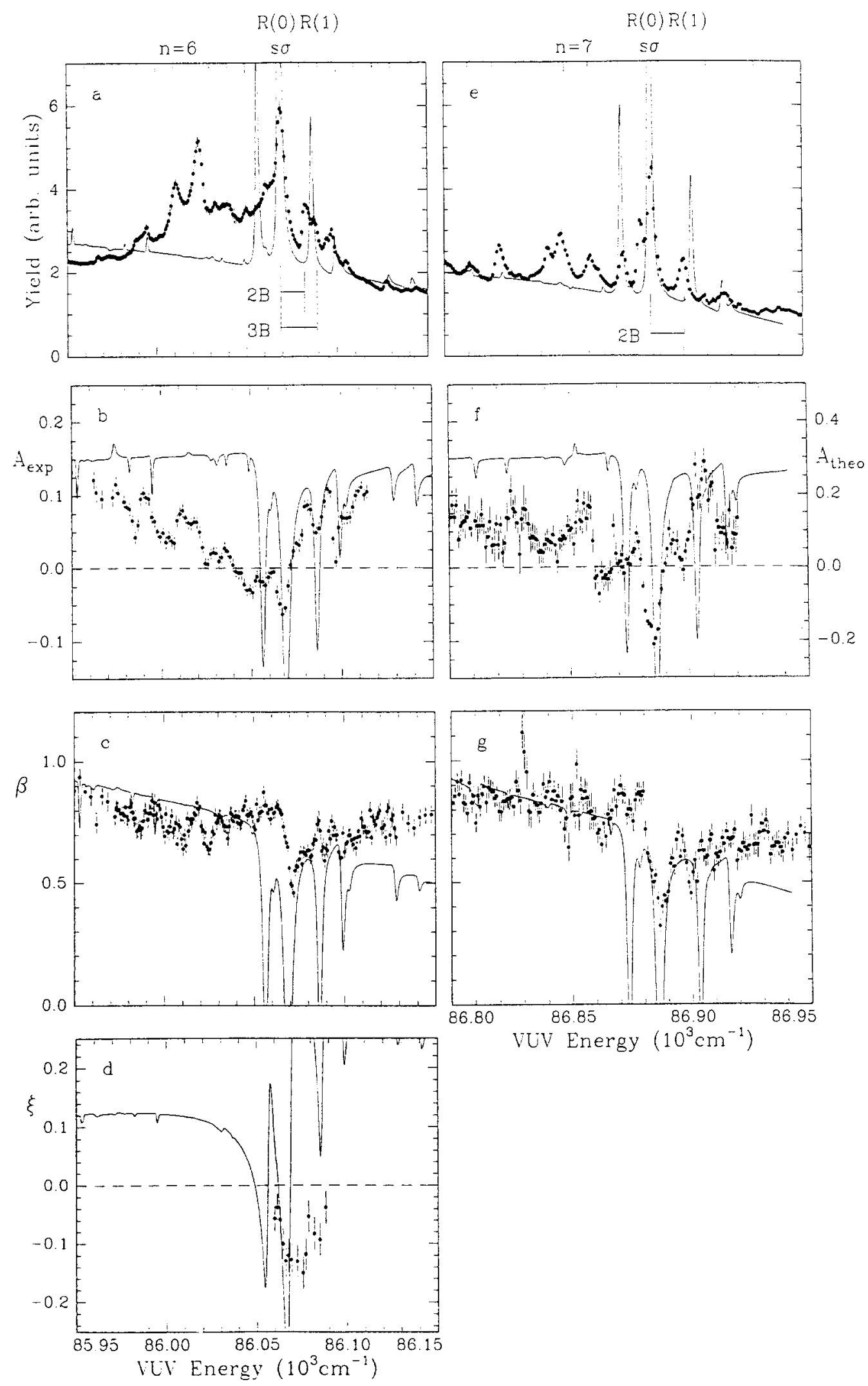

VUV Energy $\left(10^{3} \mathrm{~cm}^{-1}\right)$

Fig. 7a-g. Enlargement of the experimental results in the region of the $n=6$ and $n=7, s \sigma$ transitions. The experimental results are denoted by points with error bars, the results of the ab-initio calculation by the full lines. Note, that again the theoretical results for the $A$ parameter are given on a different scale 
perimental data have a full width at half maximum of $\approx 0.8 \mathrm{~cm}^{-1}$. With the notable exception of the strong positive resonance in the $A$ parameter at $86300 \mathrm{~cm}^{-1}$, which will be discussed later, nearly all the resonances seem to follow the predicted direction:

Generally, the $A$ parameter has a positive value, while the sharp resonances in the $s \sigma$-complex are to negative values. The overall magnitude of the $A$ parameter is lower by about a factor of two in the experiment. Missing from the experimental $A$ parameter is the broad, negative structure associated with the Fano profile of the $d \lambda$-complex at the position predicted by the calculations. These facts could be related, as will be discussed later. The weak minimum in the $A$ parameter at $\approx 86550 \mathrm{~cm}^{-1}$ could be due to either the Fano profile of the $n=7 d \delta, d \pi$ resonance or due to the $p \sigma$ resonance at this excitation energy. This influence of the Fano resonance profile can be clearly observed in the $\beta$-parameter, although the energetic position is different in experiment $\left(\approx 86600 \mathrm{~cm}^{-1}\right)$ and theory $\left(\approx 86350 \mathrm{~cm}^{-1}\right)$. The average value of the $\beta$ parameter is measured to be quite near the theoretical prediction, but again the sharp structures are not as pronounced as in theory. The differences in the results for the $\beta$-parameter presented in this paper and in references 9 and 30 reflect the different rotational temperatures of the target molecules. The first data set was obtained with an effusive beam source, yielding a sample close to room temperature. More recent measurements showed that the rotational temperature associated with the second data set was actually $\approx 30 \mathrm{~K}$ and not below $20 \mathrm{~K}$ as stated in [30]. Due to the limited number of experimental results for the parameters $\xi$ and $\alpha$, a more generalized comparison with the theoretical prediction is not possible at this time, but it seems that for both parameters the overall predictions are of the correct magnitude. In order to gain some insight into the influence of the molecular rotation onto the dynamics of the photoionization process, the rotational structure of the $s \sigma$-resonance in $n^{*}=6$ and $n^{*}=7$ will be discussed in the following section:

In Fig. 7, the results in the energy regions around $86060 \mathrm{~cm}^{-1}$ and $86880 \mathrm{~cm}^{-1}$ are presented on an enlarged scale. It is quite obvious that all observed parameters exhibit structure with a spacing in energy characteristic for rotational excitation. In Hund's case $(c)$ coupling, the spacing of successive rotational transitions is given by [43]:

$\Delta E_{\mathrm{rot}}=B^{\prime \prime} J^{\prime \prime}\left(J^{\prime \prime}+1\right)-B^{\prime} J^{\prime}\left(J^{\prime}+1\right)$

where $B^{\prime}$ and $J^{\prime}$ and $B^{\prime \prime}$ and $J^{\prime \prime}$ are the rotational constants and the angular momenta of the final and initial states, respectively. The rotational constants for the ground state of the neutral HI molecule and the molecular ion are nearly identical. Therefore, the spacing between successive rotational transitions is of the order of $2 B$. Since only the levels with $J^{\prime \prime}=0$ and $J^{\prime \prime}=1$ of the molecular ground state have appreciable population, we expect to observe $Q(1), R(0)$, and $R(1)$ transitions only. The results for $n^{*}=7$ clearly confirm this pattern, with only one line belonging to a different electronic state adjacent to the $R(0)$ transition.
In contrast, the theoretical prediction shows a separation of $3 B^{+}$between the $R(0)$ and $R(1)$ transitions. Angular momentum coupling in Hund's case $(e)$, as used in the calculation by Raseev and coworkers, gives rise to this energetic spacing [44]:

$\Delta E_{\mathrm{rot}}=B^{\prime \prime} J^{\prime \prime}\left(J^{\prime \prime}+1\right)-B^{+} J^{+}\left(J^{+}+1\right)$

where the angular momentum of the final ionic state, $J^{+}$ has half-integer values in the case of the ${ }^{2} \Pi$ ionic ground state of HI. The introduction of case $(c)$ coupling into the calculation could improve the comparison of the theoretical results with the experimental ones considerably. Both the $A$ and the $\beta$ parameter exhibit a pronounced resonance structure correlated with the resonances in the electron intensity. For all these transitions, a trend to negative values in the spin polarization is observed, although it is more pronounced for the strongest $R(0)$ transition. The unidentified state at the low-energy side of the $R(0)$ line also shows a pronounced negative structure.

In the theory which includes molecular rotation explicitly, the dependence of the total spin-polarization on the transition dipole-matrix elements is rather complicated and even includes a dependence on the continuum phase shift difference [20]. Here, we will discuss the general structure in terms of the theory developed by Cherepkov several years earlier [45]. This theory uses a Hund's case $(a)$ and $(c)$ framework. Due to angular momentum selection rules a restriction applies for $\lambda$, the projection of the angular momentum of the photoelectron on the intermolecular axis:

$\lambda=0,1,2$.

Strictly, this theory is only valid in the case where the molecular rotation is not resolved. The formula given by Cherepkov for the spin polarization parameter $A$ is:

$A=\frac{1}{2} \frac{\sum_{\ell} d_{\ell 2}^{2}-\sum_{\ell} d_{\ell 0}^{2}}{\sum_{\ell} d_{\ell 0}^{2}+\sum_{\ell} d_{\ell 1}^{2}+\sum_{\ell} d_{\ell 2}^{2}}$

where $d_{p \lambda}$ are the transition dipole-matrix elements [45]. The experimental result of a negative resonance structure is consistent with the assignment as a $s \sigma$-resonance. Each transition to a different rotational level of the same electronic state seems to have the same influence on the spin polarization. The magnitude of the effect depends on the overall line strength in the rotational transition as compared to the continuum. A similar effect arises for the angular distribution asymmetry parameter $\beta$. Due to the higher complexity of the dependence of $\beta$ on the matrix elements and the explicit dependence of $\beta$ on the phase shift differences even in the rotationless case, a discussion would be much more involved. A trend to a $\beta$ value of zero, as seen in the resonances, would be expected from a dominant $s$-wave contribution, which is spherically symmetric.

In the $n^{*}=6$ Rydberg order discussed previously [11], the pattern is more complicated. At the position of the 
$R(1)$ transition, a doublet structure is observed with a spacing of $2 B$ and $3 B$ from the $R(0)$ line. At the expected position of the $Q(1)$ transition, no structure is observed. Directly in the red wing of the $R(0)$ line, another resonance can be observed as a shoulder. The overall structure in both $A$ and $\beta$ is much less pronounced than in the cross section. The $R(0)$ line clearly gives a negative contribution, but the interpretation of the $R(1)$ structure is more involved. At the first glance, it seems that the part of the doublet at $2 B$ is associated with a positive structure in the spin polarization, whereas the structure at $3 B$ has a negative spin polarization. This would imply, that either there is a change in sign of the spin polarization for different rotational transitions of the same electronic state, or that the angular momentum coupling has to be described in Hund's case (e). Both conclusions seem to be unlikely. From the structure in $n^{*}=7$, an angular momentum coupling in case (c) can be deduced for that Rydberg order. Previously, the change to case $(e)$ coupling was found to take place between $n^{*}=10$ to $n^{*}=14$ [16]. It is highly unlikely, that $n^{*}=6$ at lower excitation energies should couple already in case $(e)$ and then switch back to case $(c)$. A change in sign does not seem to be very likely either, since in the seventh Rydberg order all rotational lines show the same behavior. Upon further consideration, the question remains how high the level of spin polarization is, which is caused by all other contributions apart from the $s \sigma$-resonance in the vicinity of the resonance structure. It could very well be that both lines are negative against an even higher "background" level. In any case, it seems that the $n^{*}=6, s \sigma$-resonance is highly perturbed. The angular asymmetry parameter $\beta$ exhibits the same complicated fine structure as the spin polarization parameter $A$. To assist in the interpretation of the complicated structure, the spin polarization component perpendicular to the reaction plane characterized by the parameter $\xi$ has been measured. Contrary to the other results, this parameter shows much broader features, which cannot be attributed to the different rotational lines quite as easily. The $\xi$ parameter depends more directly on the continuum phase shift differences than the other parameters, and does not depend on the squares of the dipole matrix elements at all. This result therefore seems to indicate a stronger influence on the phase shift in this energy region.

More insight on the nature of the perturbation can be gained by exploiting (19). Combining the results for the intensity and the spin-polarization parameter $A$, three linear combinations can be formed [18]:

$$
\begin{aligned}
& \sigma^{*}(0.5+A)=\sum_{\ell} d_{\ell 2}^{2}+\frac{1}{2} \sum_{\ell} d_{\ell 1}^{2} \\
& \sigma^{*}(0.5-A)=\sum_{\ell} d_{\ell 0}^{2}+\frac{1}{2} \sum_{\ell} d_{\ell 1}^{2} \\
& 2 \sigma^{*} A=\sum_{\ell} d_{\ell 2}^{2}-\sum_{\ell} d_{\ell 0}^{2}
\end{aligned}
$$

Only two of these are linearly independent. Since $\ell$ is not a good quantum number in molecules, it is advantageous to discuss the results in terms of $\lambda$, the projection of $\ell$
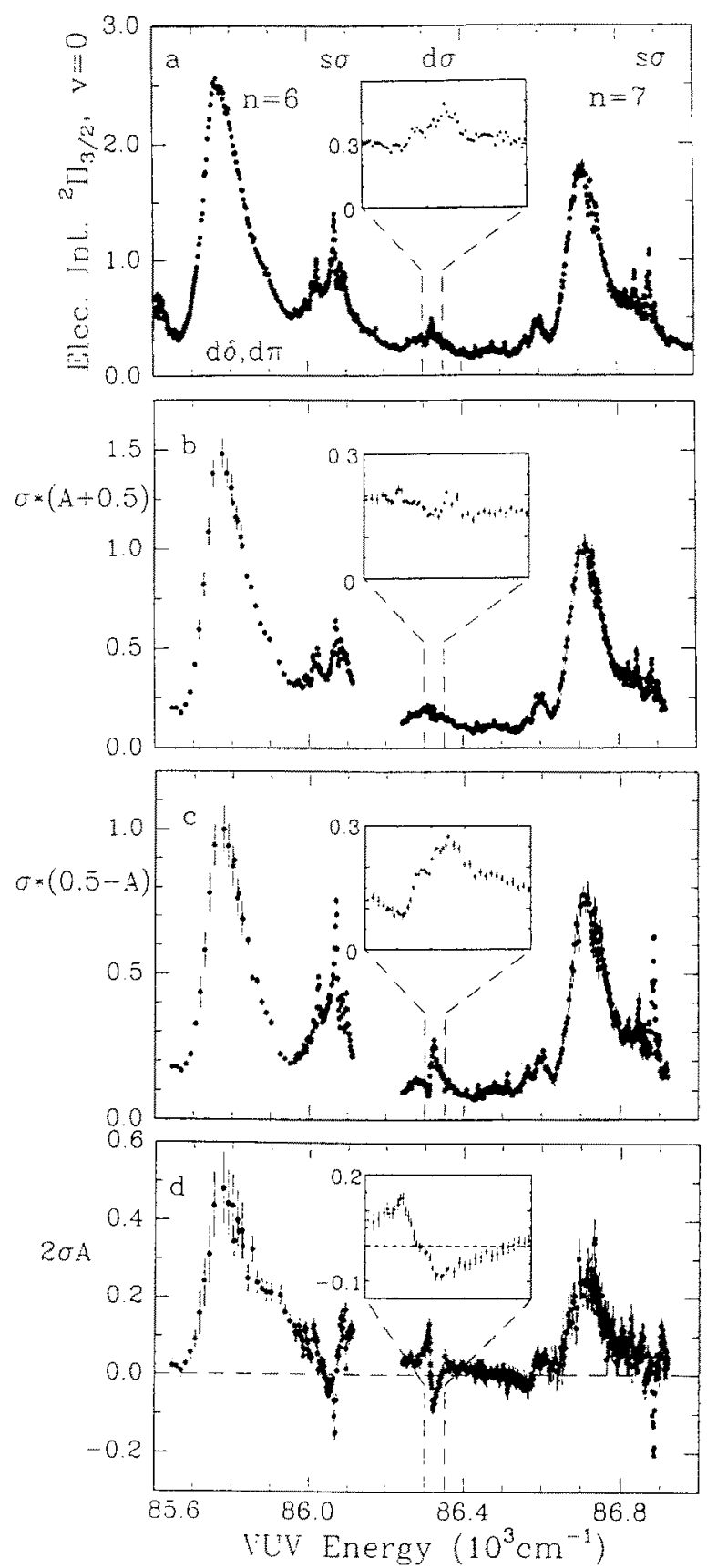

Fig. 8a-d. Comparison of the electron intensity in the ${ }^{2} \Pi_{3 / 2}, v=0$ channel with different linear combinations of cross section and angle-integrated photoelectron spin polarization according to (20)-(22); the results are denoted by points with error bars

onto the molecular axis. Only this quantum number is a good one. The advantage of using these linear combinations lies in their properties concerning their composition in $\lambda$ : Each has one $\lambda$ channel missing. A resonance with a $\sigma$-character should appear only for the contribution given in (21) and (22), but not for the one of (20).

The results for these combinations are depicted in Fig. 8. For an easier orientation, the results for the intensity are included in panel $a$. The data of Fig. $8 \mathrm{~b}$ and $c$ represent a partitioning of the total cross section into two parts with the help of the spin polarization parameter $A$. This partitioning can be done independent of a model; 
only the interpretation (right hand sides of Eqs. 20-22) depends on the particular model chosen. Surprisingly, the resonance classified as $s \sigma$ can be seen in all linear combinations for both $n^{*}=6$ and $n^{*}=7$. Two interpretations are possible: The resonance could be a complex mixture of states with different $\lambda$. In molecules, one usually finds a mixture of different $\ell$ states with the same $\lambda$ ( $\ell$-mixing) [46], and the nature of that effect is quite clear, since $\ell$ is not a good quantum number. We are not aware of a perturbation that would mix states of different $\lambda$. On the
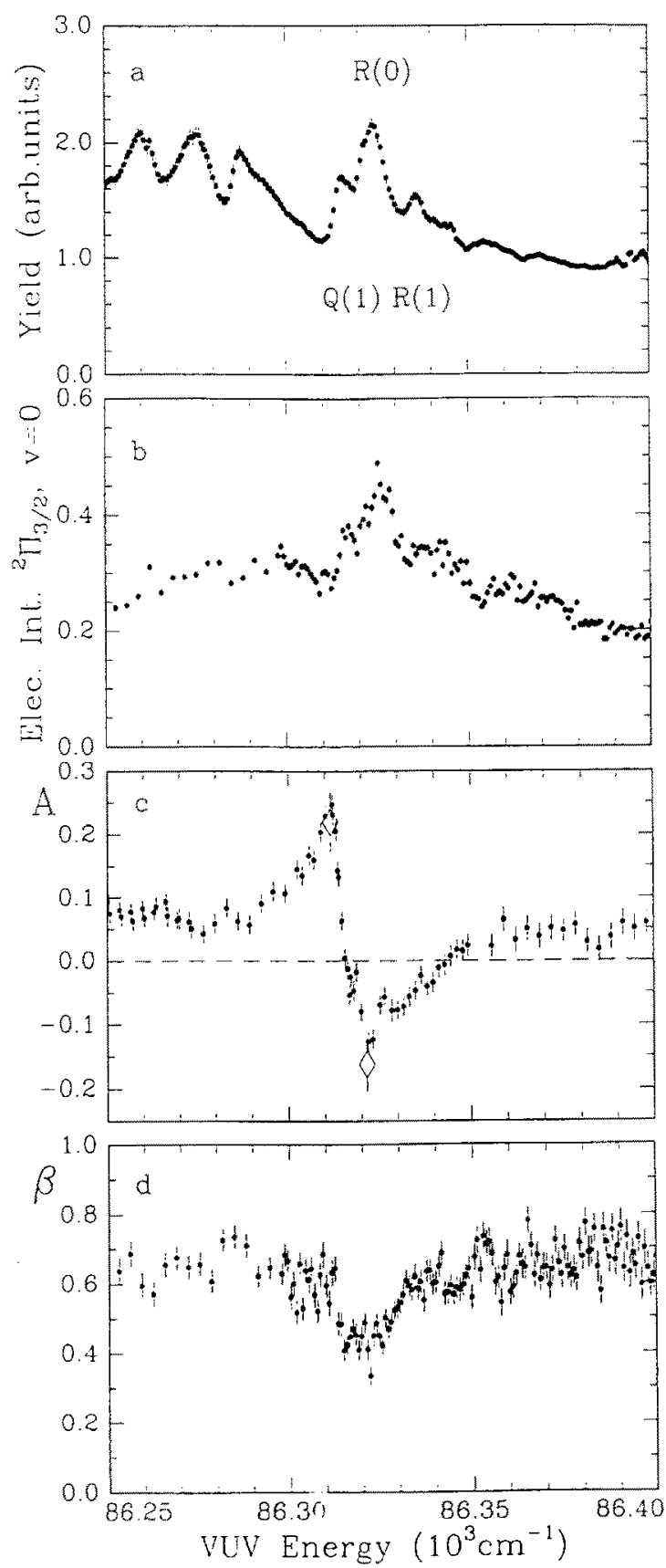

Fig. 9a-d. Enlargement of the experimental results around the $n=6$, $d \sigma$ resonance at $86323 \mathrm{~cm}^{-1}$. In a, the total photoelectron yield signal is depicted, whereas in $\mathbf{b}$, the results for the ${ }^{2} \Pi_{3 / 2}, v=0$ channel are given. The results of the spin polarization parameter $A$ were obtained with the extraction field, thereby summing over all ionic channels. In $c$, two points obtained for the ${ }^{2} \Pi_{3 / 2}, v=0$ channel are included other hand, the model of Cherepkov [45] was developed for the case of unresolved molecular rotation, and it might be insufficient to describe the behavior in this case. Here, it would be interesting to extend the model of Raseev and coworkers, which explicitly includes rotation, to Hund's case $(c)$ coupling. In Fig. 8, these linear combinations and the electron intensities are shown for all the available data in the energy region from $85600 \mathrm{~cm}^{-1}$ to $86900 \mathrm{~cm}^{-1}$. Inspection of the $d \sigma$-resonance at $86320 \mathrm{~cm}^{-1}$, which is shown for clarity again in the insets, reveals that this resonance is indeed of $\sigma$-character, since it is missing in Fig. $8 \mathrm{~b}$. This structure is of special interest, since it is has the most pronounced resonance behavior in the spin-polarization parameter $A$ of all experimental data. An enlargement of the experimental results in this region is given in Fig. 9. Here, the top panel gives the total photoelectron yield signal, which is integrated over all photoelectron emission energies. For comparison, the result of the photoelectron intensity in just the ${ }^{2} \Pi_{3 / 2}, v=0$ final ionic state is depicted in Fig. $9 \mathrm{~b}$. The data for the spin-polarization parameter $A$ in Fig. 9c were obtained with the electrostatic extraction field and discussed in part previously [21]. Included in Fig. 9c are two data points (squares) for this parameter obtained with the electron spectrometer set to the ${ }^{2} \Pi_{3 / 2}, v=0$ channel. In Fig. 9d, the results for the angular asymmetry parameter $\beta$ of the electron intensity in the ${ }^{2} \Pi_{3 / 2}, v=0$ final ionic state are shown. Since the kinetic energy of the photoelectrons associated with the ${ }^{2} \Pi_{3 / 2}, v=1$ final ionic state is less than $60 \mathrm{meV}$, it was not possible to detect the photoelectrons for this final state. A comparison of the intensity results for the two different final vibrational states reveals that the structure centered around $86270 \mathrm{~cm}^{-1}$ belongs to the excited vibrational product. This leaves the sequence of four irregularly spaced peaks to be assigned to the $d \sigma$-resonance. None of the energetic spacings observed fits into either Hund's coupling model, all the differences in excitation energy are too small. Nevertheless, it is most likely that the strongest line at $86325 \mathrm{~cm}^{-1}$ is the $R(0)$ transition, with all the other assignments given in Fig. 9 being very tentative. The spin polarization parameter $A$ shows a steep resonance feature just on the low energy side of the assigned $Q(1)$ line. To get an estimate for the influence of the vibrationally excited ionic channel, the two extreme points of this feature have been measured for the $v=0$ channel only. Within the experimental error, there is no difference detectable between these two points and the energy-integrated results. Thus, the resonance feature at around $86270 \mathrm{~cm}^{-1}$, which belongs to the vibrationally excited channel has no significant influence on the total spin polarization.

This leaves the cause for the pronounced resonance structure in the spin polarization an open question. The peak at negative values is clearly dominated by the $R(0)$ line. This behavior is to be expected for $\sigma$-type states, as has been discussed before. It is highly unlikely that the positive peak represents the 'true background', since the spin polarization levels off at a value of 0.1 at a separation of $50 \mathrm{~cm}^{-1}$ to either side of this resonance feature. It is interesting to note, that the angular asymmetry parameter 
$\beta$ has only a modest resonance structure to negative values for both the $Q(1)$ and the $R(0)$ transition. Clearly, more refined theories are needed to understand this behavior.

Finally, we would like to discuss the results for the two broad $d \delta, d \pi$-resonances with $n^{*}=6$ and $n^{*}=7$ in more detail. For these resonances, all the dynamical parameters of the photoionization process have been determined. In Fig. 6, these values have been compared to the results of the calculation by Raseev and coworkers [21], which explicitly includes molecular rotation. During the remainder of this discussion, we will not use this model for comparison. Comparing the experimental results with the calculated intensity, it is obvious, that the shape of the resonant contributions at these excitation energies is considerably different. The theoretical prediction including rotation is very similar to the results of an earlier calculation not including rotation [19]. Obviously, the electronic part of the numerical calculation does not fully represent the experimental results. The rotational fine structure has no visible influence. This can be understood since the broadening of the rotational lines due to lifetime effects is larger by more than one order of magnitude than the distance between successive rotational lines. Therefore, we will discuss these results in terms of the theory developed by Cherepkov [45].

As described above, the photoionization cross section is partitioned into components with different $\lambda$. The three possible linear combinations are not independent, therefore it is not possible to single out contributions from just one of the three $\lambda$-channels. Comparing the total cross section with the results for the three linear combinations, one finds that only the results according to (22) have a different appearance in the region of the $d \delta, d \pi$ resonance. In this combination, the contributions from the $\varepsilon \pi$-channel are eliminated. Therefore, we conclude that the dominant structure has $\pi$-character. Angular momentum selection rules would allow several $\varepsilon \pi$-channels with different value of $\ell$. But the $\ell=1$ channel is already assigned, and all channels with $\ell>2$ are expected to have quantum defects on the order of zero [19]. Therefore, the channel that is dominant here must be $\ell=2$, hence $d \pi$. This conclusion would explain the differences between the numerical calculation by Raseev and coworkers and the experimental results, since in the calculation, the $d \delta$ wave is the dominant contribution [47]. The difference in the 'background' level of the spin polarization parameter $A$ between the experimental and the theoretical results can be attributed to this problem, too. A stronger $d \pi$-wave would diminish the overall level of the $A$ parameter, since the $\lambda=1$ contribution appears only in the denominator. Comparing the results for $A$ and $\beta$, one finds that the pronounced Fano-type resonance profile that can be seen in the $\beta$-parameter is only very weak in the $A$ parameter. Again, this supports the interpretation that the $d \pi$-channel is dominating the spectrum at these energies.

The equations for the dependence of the dynamical parameters on the dipole-matrix elements and the phaseshift differences are listed in [45]. The equation for the parameter $\alpha$ can be derived by using the general formula given by Raseev et al. [48]. In principle, these formulas contain an unlimited number of dipole-matrix elements. Even restricting the dominating contributions to $\ell \leqq 2$ limits the number of independent variables only to 10,6 dipole matrix elements and 4 continuum phase-shift differences. The photoionization starts in a non-bonding $\pi$-orbital, which is strongly localized on the iodine atom [49]. The strong atomic character of the photoionization manifests itself in very weak $p$-channels, which are not allowed in the united atom. The overall appearance of the spectrum is very reminiscent of the xenon photoionization in the region between the ${ }^{2} P$-thresholds. Thiel argues that in the case of non-resonant $\pi$-ionization, partial waves with $\ell \geqq 3$ can be neglected if the kinetic energy of the photoelectron is larger than $10 \mathrm{eV}$ [50]. In the photoionization of $\mathrm{HI}$ in the spin-orbit region, the typical kinetic energy of the photoelectron is only $250 \mathrm{meV}$. On the other hand, the autoionization spectrum is dominated by resonant contributions. In the spectral region of interest, the important contributions are in the $d$-channel, therefore this restriction could be justified. With only five observables, it is impossible to determine 10 independent variables. Further simplifications have to be made. One would be to sum all $\ell$ contributions to each $\lambda$. Angular momentum selection rules allow only $\lambda=0,1$, and 2 . Then, only five variables will remain, three dipole-matrix elements and two phase-shift differences. This is not possible with the formalism of Cherepkov [51]. Another simplification would be to disregard the $\ell=1$ channels, which are of minor importance at these photon energies. This would result in four matrix elements. The phase-shift differences between waves of same $\ell$ but different $\lambda$ should be negligible, too. Therefore, only one phase-shift difference remains, between the $\ell=2$ and the $\ell=0$ channel.

We tried to fit our experimental results to this model, using various non-linear least-squares fitting procedures [52]. As a result, we found at least six different solutions to the problem. Of these, there were several, which would support the interpretation of a dominating $d \pi$-wave. But we also found more than one combination with a dominating $d \sigma$-channel. A detailed energy dependence of all dynamical parameters in this region might help in the interpretation of the results, as it has been used before in the study of atomic photoionization [32]. At this point in time, we do not feel it is justified to single out one of the solutions as the correct one, even though there is supporting evidence for a strong amplitude of the $d \pi$ wave.

\section{Conclusions}

The experiments discussed in this paper have demonstrated the feasibility of angle- and spin-resolved photoelectron spectroscopy in conjunction with narrow bandwidth excitation for the study of molecular systems. The data obtained in these initial experiments on the $\mathrm{HI}$ molecule provided for a deeper insight into the detailed dynamics of the photoionization process. The influence of the molecular rotation on the dynamical photoionization parameters was observed by combining a jet-cooled 
molecular beam, where only the lowest rotational levels are populated, and high-resolution VUV radiation with angle- and spin-resolved photoelectron spectroscopy. In comparison to the calculation of [21] similar general trends were found in the experimental data, but quantitative agreement could not be obtained, neither with respect to the magnitude nor the width of the rotational features in the dynamical parameters. The results indicate that Hund's case $(c)$ is the most appropriate coupling case to describe the recorded data in low Rydberg orders. In general, the different parameters probe different parts of the transition moments. Therefore, the polarization data gave additional information on the outgoing partial waves when the results for $\sigma$ and $A$ were combined. The results for the additional parameters determined in angleresolved measurements did not lead to further, unambiguous interpretations. They probe not only the transition amplitudes but also the phase shift differences of the outgoing partial waves. For a thorough understanding of the experimental results, it is highly desirable to obtain better theoretical models of the underlying processes.

One problem in interpreting the results of the angleand spin-resolved molecular photoelectron spectroscopy is the large number of theoretical quantities needed as opposed to the limited number of experimental observables available. In a recent REMPI experiment on NO, Zare and coworkers [53] have demonstrated, that under certain circumstances, it is possible to restrict the number of variables so that all quantum mechanical parameters can be calculated from experimental results obtained without analysis of the electron spin polarization. The very special conditions which were necessary for that experiment prohibit a generalization of the scheme. Observing the spin-polarization of the emitted photoelectrons is a more general method. If it were possible to do so with rotational resolution in the kinetic energy of the photoelectron, the number of variables needed to describe the experiment would be more limited.

The authors would like to thank M. Büchner and A. Elizarov for their assistance in obtaining the experimental results and $T$. HuthFehre for help in the design of the experimental apparatus. We would also like to thank G. Raseev, N.A. Cherepkov and $H$. Lefebvre-Brion for the numerous discussions on the subject. Financial support from the Deutsche Forschungsgemeinschaft (SFB 216) and the European Community is gratefully acknowledged.

\section{References}

1. Starace, A.F.: In: Handbuch der Physik, Vol. 31. Mehlhorn, W. (ed.), p. 11. Berlin, Heidelberg, New York: Springer 1983

2. Pratt, S.T., Dehmer, P.M., Dehmer, J.L.: In: Advances in multiphoton processes and spectroscopy, Vol. 4. Lin, S.H. (ed.), p. 69. Singapore: World Scientific 1988

3. Vidal, C.R.: In: Topics in Applied Physics, Vol. 59. Tunable lasers. Mollenhauer, L.F., White, J.C. (eds.), p. 57. Berlin, Heidelberg, New York: Springer 1987; Shen, Y.R.: In: Non linear infrared generation. Shen, Y.R. (ed.), p.1. Berlin, Heidelberg, New York: Springer 1986

4. Hilbig, R., Hilber, G., Lago, A., Wolff, B., Wallenstein, R.: Comm. At. Mol. Phys. 18, 157 (1986)

5. Müller-Dethelfs, K., Schlag, E.W.: Annu. Rev. Phys. Chem. 42, 109 (1991)
6. Asbrink, L.: Chem. Phys. Lett. 7, 549 (1970)

7. Heinzmann, U.: In: Electronic and atomic collisions. Lorentz, D.C., Meyerhof, W.E., Peterson, J.R. (eds.), p. 37. Amsterdam: Elsevier 1986

8. Heinzmann, U.: J. Phys. B13, 4353, 4867 (1980)

9. Mank, A., Drescher, M., Huth-Fehre, T., Schönhense, G. Böwering, N., Heinzmann, U.: J. Phys. B22, L487 (1989)

10. Tonkyn, R.G., Wiedemann, R.T., White, M.G.: J. Chem. Phys. 91, 6632 (1989)

11. Huth-Fehre, T., Mank, A., Drescher, M., Böwering, N., Heinzmann, U.: Phys. Rev. Lett. 64, 396 (1990)

12. Hart, D.J., Hepburn, J.W.: Chem. Phys. 129, 51 (1989)

13. Mank, A.: Ph.D. Thesis, University of Bielefeld 1991 (unpublished)

14. Hilbig, R., Wallenstein, R.: IEEE J. of Quantum Electronics QE-19, 1759 (1983)

15. Huber, K.P., Herzberg, G.: Molecular spectra and molecular structure IV: constants of diatomic molecules. New York: Van Nostrand 1979

16. Mank, A., Drescher, M., Huth-Fehre, T., Böwering, N., Heinzmann, U., Lefebvre-Brion, H.: J. Chem. Phys. 95, 1676 (1991)

17. Böwering, N., Müller, M., Salzmann, M., Heinzmann, U.: J. Phys. B24, 4793 (1991)

18. Böwering, N., Salzmann, M., Müller, M., Klausing, H.W., Heinzmann, U.: Phys. Rev. A45, R11 (1992)

19. Lefebvre-Brion, H., Giusti-Suzor, A., Raseev, G.: J. Chem. Phys. 83, 1557 (1985)

20. Raseev, G., Cherepkov, N.A.: Phys, Rev. A42, 3948 (1990)

21. Büchner, M., Raseev, G., Cherepkov, N.A.: J. Chem. Phys. 96, 2691 (1992)

22. Huth-Fehre, T., Mank, A., Drescher, M., Böwering, N., Heinzmann, U.: Phys. Scr. 41, 454 (1990)

23. Hunter, W.R.: Appl. Opt. 17, 1259 (1978)

24. Rabinovitch, K., Canfield, L.R., Madden, R.P.: Appl. Opt. 4, 1005 (1965)

25. Schönhense, G.: Phys. Rev. Lett. 44, 640 (1980)

26. Bey, P.P., Giuliani, J.F., Rabin, H.: Phys. Lett. 26A, 128 (1968)

27. Heinzmann, U.: J. Phys. B11, 399 (1978)

28. Jost, K.: J. Phys. E12, 1006 (1979)

29. Böwering, N., Klausing, H.W., Müller, M., Salzmann, M., Heinzmann, U.: Chem. Phys. Lett. 189, 467 (1992)

30. Mank, A., Drescher, M., Huth-Fehre, T., Schönhense, G., Böwering, N., Heinzmann, U.: J. Electron Spectr. Related Phenom. 52, 661 (1990)

31. Berkowitz, J.: Photoabsorption, photoionization and photoelectron spectroscopy, pp. 358ff. New York: Academic Press 1979

32. Lee, C.M.: Phys. Rev. A10, 1598 (1974)

33. Keßler, J.: Polarized electrons. Berlin, Heidelberg, New York: Springer 1985

34. Heckenkamp, Ch., Schäfers, F., Schönhense, G., Heinzmann, U.: Z. Phys. D2, 257 (1986)

35. Heckenkamp, Ch., Eyers, A., Schäfers, F., Schönhense, G., Heinzmann, U.: Nucl. Instrum. Methods A246, 500 (1987)

36. Huth-Fehre, T.: Ph.D. Thesis, University of Bielefeld 1989 (unpublished)

37. Born, M., Wolf, E.: Principles of optics. p. 30fr. Oxford: Pergamon Press 1980

38. Heinzmann, U.: J. Phys. E10, 1001 (1977)

39. Huang, K.H.: Phys. Rev. A22, 223 (1980)

40. Cherepkov, N.A.: Adv. At. Mol. Phys. 19, 395 (1983)

41. Carlson, T.A., Gerard, P., Krause, M.O., Wald, G. von, Taylor, J.W., Grimm, F.A.: J. Chem. Phys. 84, 4755 (1988)

42. Eland, J.H.D., Berkowitz, J.: J. Chem. Phys. 67, 5034 (1977)

43. Herzberg, G.: Molecular spectra and molecular structure I. Spectra of diatomic molecules. New York: Van Nostrand 1950 44. Lefebvre-Brion, H.: J. Chem. Phys. 93, 5898 (1990)

45. Cherepkov, N.A.: J. Phys. B14, 2165 (1981)

46. Lefebvre-Brion, H., Field, R.W.: Perturbations in the spectra of diatomic molecules. Orlando: Academic Press 1986 
47. Raseev, G.: Private communication (1991)

48. Raseev, G., Keller, F., Lefebvre-Brion, H.: Phys. Rev. A36, 4759 (1987)

49. Lempka, H.J., Passmore, T.R., Price, W.C.: Proc. Roy. Soc. A304, 53 (1968)

50. Thiel, W.: Chem. Phys. 77, 103 (1983)
51. Cherepkov, N.A.: Private communication (1992)

52. Press, W.H., Flannery, B.P., Teukolsky, S.A., Vetterling, W.T.: Numerical recipes. Cambridge: Cambridge University Press 1986 53. Reid, K.L., Leahy, D.J., Zare, R.N.: Phys. Rev. Lett. 68, 3527 (1992) 